Gait \& Posture

Original Article

\title{
A cross-sectional study of age-related changes in plantar pressure distribution between 4-7 years: a comparison of regional and pixel- level analyses
}

\author{
Jill Phethean ${ }^{1}$, Todd C Pataky ${ }^{2 *}$, Christopher J. Nester ${ }^{1}$, Andrew H. Findlow ${ }^{1}$ \\ ${ }^{*}$ Corresponding author \\ ${ }^{1}$ School of Health Sciences, University of Salford, Manchester, M6 6PU, UK \\ ${ }^{2}$ Department of Bioengineering, Shinshu University, Tokida 3-15-1 Ueda, Nagano, 386-8567, Japan
}

Corresponding author contact details:

Todd Pataky, Ph.D.

Shinshu University, Department of Bioengineering

Nagano-ken, Ueda-shi, Tokida 3-15-1 Japan 386-8567

email: tpataky@shinshu-u.ac.jp

Acknowledgements-None. 


\section{Abstract}

Quantifying morphological and functional development in children's feet, and thereby establishing development norms is difficult. In addition to practical challenges of experimentation on children, measurement equipment like plantar pressure (PP) platforms are almost exclusively geared toward adult-sized feet. These PP quantification problems may be exacerbated by typical regional data analysis techniques, which further reduce spatial resolution. The goal of this study was to quantify PP distributions in developing children, and also to compare the results obtained from typical (regional) techniques with those obtained from a higherresolution (pixel-level) technique. Ninety-eight children between four and seven years of age were assessed in a cross-sectional design. Maximum PP distributions were collected for each child, and these pressures were linearly regressed against age in days. Present results agree with previous investigations in that maximum pressures and maximum pressure changes occurred in the forefoot. However, results from the present pixel-level technique suggest that these changes are limited to the central metatarsals, and that regional methods can suggest significance where none exists in the actual raw (pixel-level) data, due to signal aliasing and, in particular, to conflation of regional boundaries. We postulate that increased central metatarsal pressures are reflective of the coupling between generalized joint laxity decreases and relatively increasingly inclined central metatarsal bones with age.

Keywords - Foot morphology; Walking; Gait; Morphological and functional development; Children; Statistical parametric mapping 
Research Highlights

- We examined age-related plantar pressure changes in young children

- Regional pressures increased with age at the central metatarsals and medial midfoot

- Pixel data disagreed with regional midfoot and heel data and were also more detailed

- Pixel data showed that children's feet tend to become longer and narrower with age

- Pixel-level analysis overcomes regional analysis' inadequate spatial resolution 


\section{Introduction}

Plantar pressure (PP) distributions can be used to characterise foot function and have been shown to change during normal development towards adulthood. For example, several studies report that maximum $\mathrm{PP}\left(\mathrm{PP}_{\max }\right)$ increases under the total foot, the hindfoot and the forefoot between the commencement of independent walking and 13 years of age, but not under the midfoot [1-5]. Age-specific foot loading patterns for toddlers demonstrate the highest $\mathrm{PP}_{\max }$ under the hallux; whereas in 7 year olds the highest $\mathrm{PP}_{\max }$ is under the hindfoot [2,5]. These clearly identified foot loading patterns are considered to be part of the normal developmental stages of the growing foot $[1,4,6]$.

Whilst there are child-specific variations in the precise timing of growth milestones, the period between four and seven years of age demonstrates considerable neuromuscular and skeletal change [7-9], including changes in foot structure, morphology and function $[3,5,10,11]$. This period spans the transition from immature to mature gait [12] and changes in bone length and structural composition occur alongside rotational changes in the transverse and frontal planes $[7,8,10,11,13]$. Changes in gait are seen in the temporal-spatial parameters, and in kinematics, electromyography and kinetics related to ankle joint motion $[12,14,15]$. Consequently, the changes occurring in this four to seven year old period suggest potential differences in the foot loading patterns within this period.

There are two potential problems with work to-date that both stem from potentially inadequate spatial (anatomical) resolution. This first is that the midfoot and forefoot were previously reported as whole regions of interest (ROI) $[3,5]$, rather than with 
mediolateral subdivisions. This is a potential problem because we know that the adult foot demonstrates differences in the mobility of the medial and lateral midfoot and forefoot [16]. Thus smaller ROIs, and their more finely detailed anatomical information, may allow us to draw more definitive conclusions regarding the relation between PP and the maturation of the foot's multiple structures and articulations.

The second potential problem is that previously employed ROIs may have impinged upon neighbouring anatomical structures. For example, midfoot ROI definitions typically extend posteriorly toward the anterior heel and anteriorly toward the posterior forefoot. The problem is that there is typically a large pressure increase from the midfoot to both the anterior heel and the posterior forefoot. Therefore if midfoot ROIs are not carefully defined then midfoot results could be driven by the heel or forefoot, or both. Indeed, this phenomenon has been shown to have the potential to create, eradicate, and even reverse statistical and biomechanical conclusions [17].

We presently refer to the aforementioned problems as "intra-region variation" and "inter-region conflation", respectively. The cause of both is inadequate spatial resolution in ROI data. Healthy and pathological adult PP data have spatial wavelengths on the order of $30 \mathrm{~mm}$ [18] and $20 \mathrm{~mm}$ [19], respectively. Plantar pressures must therefore be measured with (Nyquist) resolutions of at least $15 \mathrm{~mm}$ and $10 \mathrm{~mm}$, respectively, to avoid signal aliasing. Although original pixel-level data typically have adequate resolutions, on the order of $5 \mathrm{~mm}$, ROI-level data typically have resolutions on the order of $50 \mathrm{~mm}$, which are too gross. Problems associated with inadequate spatial resolution have been addressed elsewhere for adult feet 
$[18,19]$, but spatial resolution has not, to our knowledge, been previously explored for children's data.

The purpose of this study was to investigate the effect of spatial resolution on the conclusions regarding PP development amongst children aged four to seven years of age. To this end we presently compare the results of hypothesis testing on pixel-level data vs. ROI-level data. The present null hypothesis was that age has no effect on PP distribution.

\section{Methods}

\subsection{Participants}

Subsequent to approval from the ethics committee at the University of Salford 46 girls and 52 boys aged between four and seven years old were recruited from nurseries, schools, university staff and students, and by word of mouth. Although not analyzed categorically, mean ( \pm st.dev.) ages for four-, five-, six- and seven-year olds were: $48.5 \pm 1.1,60.7 \pm 1.0,72.2 \pm 0.8$ and $84.4 \pm 1.0$ months, respectively. Parents and guardians gave informed consent in all cases. Since we previously found that gender failed to predict plantar pressure differences amongst children [20] genders were presently pooled.

Children met four criteria to be included: (1) normal gestational period of 37-42 weeks; (2) correct development of locomotion skills; (3) within $0.4^{\text {th }}-99.6^{\text {th }}$ centile for height and weight in relation to their chronological age; and (4) no gross gait abnormalities during visual inspection e.g. tiptoe walking, limping, tripping, excessive in/out-toed gait. Normal attainment of locomotion included walking prior to 17 
months of age and appropriate age-related development of locomotion skills assessed by movement and balance, and stair tasks in accordance with the 'Schedule of Growing Skills' [21]. Stature and weight reference curves [22] were used to determine height and weight centiles.

\subsection{Procedure}

PP data were collected within 60 days of each child's birthday, barefoot in mid-gait at natural walking speed [23] using an optical pedobarograph system (Biokinetics Inc, Bethesda, USA; now defunct). The pressure plate surface dimensions, resolution, and sampling frequency were: $57 \mathrm{~cm} \times 48.5 \mathrm{~cm}, 3 \mathrm{~mm} \times 2 \mathrm{~mm}$, and $25 \mathrm{~Hz}$, respectively. A familiarisation period minimised targeting of the plate and determined a start position that ensured five to six steps were taken prior to plate contact. Trials were excluded if the foot failed to contact close to the plate center, and also if gait was observed to be irregular (e.g. targeting, excessive acceleration/deceleration, irregular cadence/stride length). Five trials were collected from each foot.

\subsection{Region of Interest (ROI) analysis}

Nine anatomical ROIs were manually defined on each $\mathrm{PP}_{\max }$ image: Heel, MedMF and LatMF (medial and lateral midfoot), Hallux, and MH1-5 (the five metatarsal heads). Constant-sized ellipsoids were used: $3.38 \mathrm{~cm}^{2}$ (Heel), $2.16 \mathrm{~cm}^{2}$ (MedMF and LatMF), $1.69 \mathrm{~cm}^{2}$ (Hallux and MH1), and $1.09 \mathrm{~cm}^{2}$ (MH2-MH5). The first set of ROI analyses ("Method A") merged: (1) the MF region and (2) the MH region (Fig.1a) to mimic previous developmental studies that did not sub-divide the midfoot or metatarsal regions [3,5]. The second set of analyses ("Method B") assessed the nine ROIs separately (Fig.1b). Although there was little or no foot contact in the medial 
midfoot region in some subjects, we employed a medial midfoot ROI to follow convention [24].

Maximum pressure values were extracted from each ROI, were averaged withinsubjects, and were then linearly regressed against age. The threshold for significance was set at $\alpha=0.05$, and Bonferroni corrections of 0.013 and 0.00568 were used to correct for multiple comparisons across the four and nine ROIs, respectively. The left and right feet were analysed separately for cross-validation.

\subsection{Whole-foot (pixel-level) analysis}

$\mathrm{PP}_{\max }$ distributions for each step were first registered (i.e. spatially aligned) withinsubjects and within-feet using a rapid frequency-based technique [25]. The resulting average distributions (one per-subject, per foot) were registered between-subjects using an optimal linear (scaling) transform. The chronologically first seven-year-old's feet were used as the registration templates, and then, to avoid potential bias from template peculiarities, all images were re-registered to the resulting between-subject mean. A linear transformation was utilized to retain subjects' original morphology.

At this point all images were registered to a common coordinate system, with a total of 196 mean images (one per subject, per foot). Identical to the regional data above, these images were regressed (in a pixel-wise manner) against age, yielding two linear correlation coefficient ( $r$ ) distributions, one for each foot. The $r$ distributions were then transformed into $t$ distributions via the identity:

$$
t=r(n-2)^{0.5}\left(1-r^{2}\right)^{-0.5}
$$


where $n$ is the number of subjects and $t$ has the Student's $t$ distribution with degrees of freedom: $(n-2)$. Since $t$ is presently both parametric and pixel-specific it is referred to as a 'statistical parametric map' (SPM) [26]. Statistical significance was determined topologically as the probability that a purely random (Gaussian) spatial process, with identical smoothness, will produce suprathreshold pixel-clusters of the observed size [26]; presently a threshold of $|t|>2$ was selected because this is the lowest threshold that has been shown to be valid for plantar pressure data [27]. Smoothness was estimated from the regression residuals using the average spatial gradient [26].

\section{Results}

Between-subject average pressure distributions (Fig.2) qualitatively indicate: (i) increased pressure with age, especially at the heel, central metatarsal heads, and hallux, and (ii) a tendency for the foot to become more slender with age (i.e. relatively longer and narrower). Whole-foot peak pressure averaged 258, 280, 293, and $287 \mathrm{kPa}$ (all at the Heel) for the 4-7 yearly intervals on the left (Table 1, Fig.3), with comparable values on the right (Supplementary Material).

Regression analysis of the Method A ROI data (Fig.1a) reached significance only for the $\mathrm{MH}$ region, with $r^{2}$ values of $0.007,0.001,0.077$ and 0.001 and $p$ values of 0.403 , 0.896, 0.006 and 0.861 for the Heel, MF, MH and Hallux regions, respectively. Regression analysis of the Method B ROI data (Fig.1b) yielded significant positive correlations between pressure and age in both the left (Fig.3) and right feet (Supplementary Material), but only for MH3 $(p<0.001)$ and MedMF $(p<0.005)$. 
Regression analysis of the pixel-level data (Fig.4a) echoed both aforementioned mean-image trends (Fig.2). In particular, pressures tended to be positively correlated with age at the heel, central metatarsals, and hallux. Additionally, the broad rings of negative correlation around the feet (Fig.4a) indicate that the foot tended to become more slender with age. Statistical inference (Fig.4b) confirmed that central metatarsal pressures were positively correlated with age $(p=0.002)$, but failed to find significance at either the heel or hallux. The ring of negative correlation observed around both feet reached significance only at the lateral heel in the left foot $(p=0.004)$.

\section{Discussion}

\subsection{Discrepancies amongst analysis methods in descriptions of age-related changes}

The present midfoot, metatarsals, and heel results were dependent on the analysis method employed. ROI Method A (Fig.1a) failed to find a midfoot effect, but Method B (Fig.1b) found increasing medial midfoot pressures. The former is consistent with qualitative observations of the mean PP distributions (Fig.2), but the latter is not. This apparent discrepancy is explained by inter-region conflation (see Introduction). Since the foot becomes increasingly slender with age (Fig.2; c.f. [5]), we were presently forced to draw the medial midfoot region increasingly more laterally in order to maintain a morphologically consistent mediolateral delineation. That is, the MedMF region can be drawn nearly in line with the lateral edge of the hallux at four years, but it must be positioned more laterally at seven years. Thus the medial midfoot data (Fig.3), instead of truly reflecting pressure changes with age, may actually reflect increasing conflation with the lateral midfoot. The true midfoot trend is observed most clearly at the pixel-level (Fig.4a); the pixel-level data resolve the ROI Method A-B discrepancy. 
The present study employed manual ROI definitions, so it is possible that more objective, anatomically-defined ROIs $[28,29]$ may produce more consistent results. However, it has also been shown that midfoot ROI data are less reliable than midfoot pixel-level data [30] because small changes in ROI boundary definitions can greatly affect the underlying pressure values [31]. Thus, while anatomically defined ROIs can be expected to produce more consistency, no ROI is expected to be as consistent as the pixel-level data.

All three methods found metatarsal pressure increases but the interpretations of each are different because their anatomical resolutions are different. For Method A the interpretation is simply that forefoot pressures increase with age, which echoes previous results that employed a full-forefoot ROI [3,5]. For Method B the interpretation, with an uncorrected threshold of $\mathrm{p}=0.05$, is that the three medial metatarsal heads' pressure increase with age (Fig.3), and thus that the foot is loaded increasingly medially with age. With a Bonferroni correction of $\mathrm{p}=0.00568$, the interpretation would change to the pixel-level interpretation of increased central metatarsal pressures (Fig.4b). The statistical signal clearly peaked at MH3 and tapered off medially and laterally from there; thus any region drawn medial or lateral to MH3 is driven by pressure trends on its border closest to $\mathrm{MH} 3$, where the statistical signal is strongest. This can be seen in the regional $p$ values (Fig.3): $p=0.029,0.009,0.000$, $0.117,0.992$ for MH1-5, respectively.

None of the three methods found a significant positive correlation between heel pressure and age, and only the pixel-level approach found significant negative 
correlation (Fig.4b), but only on the lateral heel periphery. This apparent discrepancy is explained by intra-region variation. The heel clearly becomes relatively thinner with age (Fig.2), a change which is reflected as a cloud of negative correlation around the low-pressure periphery of the foot (Fig.4a). However, this morphological change could not be captured with ROI data because the highest pressures (i.e. the central heel) drives the ROI results. Thus a single heel ROI has insufficient spatial resolution to discriminate amongst multiple, and indeed opposite within-heel trends. Although the pixel-level analyses presently failed to find similar evidence of morphological changes in the right foot, or at any other peripheral foot location in general, the qualitative trend suggests increasingly slenderness with age (Fig.2), and this trend is captured by the raw statistical image (Fig.4a). This increasing slenderness would be expected to reach significance with a longitudinal study because between-subject morphological variability is essentially eliminated in longitudinal analyses.

One may argue that the subjectivity of the present manual ROI definitions may have affected the results. We do not dispute this, but we'd argue that automated or otherwise algorithmic ROI definition is equally susceptible to the aforementioned risks. All ROIs can lie on sharp pressure gradients [17,31] and are thus susceptible to inter-region conflation. Furthermore, no ROI scalar can represent multiple within-ROI trends. More formally, ROI techniques risk aliasing because they sample the PP distribution with insufficient spatial resolution. It has been estimated that statistical signals in PP data have spatial wavelengths on the order of $20 \mathrm{~mm}[18,19,27]$; at this spatial frequency each ROI should have a maximum size of $10 \mathrm{~mm}$ to avoid aliasing. 
Last, we'd argue that the pixel-level approach offers two non-trivial advantages over the ROI approach. First, its spatial resolution is far-superior (Fig.4 vs. Fig.3), and its anatomical resolution is therefore also superior. Second, pixel-level results are superimposed directly on the foot (Fig.4), so whole-foot effects can be appreciated at a single glance. With the ROI results (Fig.3) it is much more difficult to appreciate how effects are distributed across the foot.

\subsection{Comparison with previous studies}

The present pressure values match well with three previous studies [2,3,5] (Table 1); we are unaware of other studies that provide data for four-seven year olds. All studies report the greatest pressure under the heel, and higher hallux than forefoot pressure. The main difference between the present and previous study is that the midfoot pressures tended to be lower. A possible explanation is conflation; the cited studies employed automated regionalization, which has been shown elsewhere to be highly susceptible to both anterior heel and posterior midfoot conflation [17,31]. It is possible that the present manually defined ROIs avoided conflation to a greater degree, but since ROI definition information was not provided in the cited studies we are unable to explore this possibility further.

The present forefoot pressures were similar to previous reports [5]. Other studies reported higher forefoot pressure for children aged five years and older [2,3]. The large inter-individual variations in the forefoot region, which were both observed presently (Fig. 3) and reported previously [5] suggest that these discrepancies may simply be an artefact of sampling. 
Finally, although we cannot compare our data directly, it is notable that significant age-related pressure increases have been previously reported for the calcaneus and hallux between the ages of one and seven [2]. The absence of similar increases in the present dataset suggests that these changes are likely to occur prior to four years of age.

\subsection{Morphological interpretation}

The present results (Figs.2,4) support previous reports that the developing foot becomes increasingly slender with age [5]. While the cited study demonstrated this morphological change explicitly via a decrease in the foot's length-width ratio from $0.4 \pm 0.02$ in four year-olds to $0.38 \pm 0.02$ in 7 year-olds, the present results are the first, to our knowledge, that report the same result implicitly, via a systematic PP distribution change, and in particular via a cloud of negative correlation around the foot periphery (Fig.4a).

\subsection{Functional interpretation}

The present results (Fig. $4 \mathrm{~b}$ ) suggest that forefoot PP increases $[2,3,5]$ are limited to the central metatarsals. It is possible that this is caused by the interaction amongst three factors: (1) generalized decreases in joint laxity with age [32], (2) increasingly inclined metatarsal bone postures, secondary to a reduction in talar head decline [13], and (3) progression towards adult foot kinematics, which involve less central metatarsal movement compared to the 1 st and 5th metatarsals $[16,33]$. Therefore, this inclined central metatarsal posture coupled with increased joint stiffness would likely increase pressures under the metatarsal heads, and the differences in the central vs. 
peripheral metatarsal postures [16,31] could explain the presently observed pressure distribution changes (Figs.2,4).

While the present study focused on maximal plantar pressures, we acknowledge that many other morphological and functional changes occur during maturation of the skeleton and gait between ages 4-7. These are not all fully described in the literature and thus it is not possible to detail how concurrent morphological and functional changes contribute to the observations in this study. However, a variety of other parameters, like center-of-pressure progression, must be considered to fully appreciate the biomechanical bases for the present results.

\section{$\underline{\text { Conclusion }}$}

The present data are broadly in line with previous investigations that report forefoot pressure increases in the developing child's foot as a function of age. These results suggest that PP increases are limited to the central metatarsals, which we speculate is caused by increased central metatarsal joint stiffness secondary to increased weightbearing demands. The present results comparing regional and pixel methods reiterate previous findings that regional data can distort the underlying PP distribution due to signal aliasing. Since pixel level techniques provide much better anatomical resolution than regional techniques, it is recommended that regionalization should be avoided when analysing PP data, especially in children where pixel sizes are comparatively large with respect to foot size. 


\section{References:}

[1] Bosch K, Gerb J, Rosebaum D. Preliminary normative values for foot loading parameters of the developing child. Gait Posture 2007; 26:238-247.

[2] Bosch K, Nagel A, Weigend L, Rosenbaum D. From first to last steps in lifepressure patterns of three generations. Clin Biomech (Bristol Avon) 2009; 24:676681.

[3] Bosch K, Gerb J, Rosebaum D. Development of healthy children's feet-Nineyear results of a longitudinal investigation of plantar loading patterns. Gait Posture $2010 ; 32(4): 564-571$.

[4] Hallemans A, D’Aout K, De Clercq D, Aerts P. Pressure distribution patterns under the feet of new walkers: The first two months of independent walking. Foot Ankle Intl 2003; 24(5); 444-453.

[5] Müller S, Carlsohn A, Müller J, Baur H, Mayer F. Static and dynamic foot characteristics in children aged 1-13 years: a cross-sectional study. Gait Posture 2012; 35(3):389-94. 
[6] Bertsch C, Unger H, Winkelmann W, Rosenbaum D. Evaluation of early walking patterns from plantar pressure distribution measurements. First year results of 42 children. Gait Posture 2004; 19(4):235-242.

[7] Anderson M, Blais M, Green WT. Growth of the normal foot during childhood and adolescence; length of the foot and interrelations of foot, stature and lower extremity as seen in serial records of children between 1-18 years of age. Am J Phys Anthrop 1956; 14(2):287-308.

[8] Salenius P, Vankka E. The development of the tibiofemoral angle in children. J Bone Joint Surg 1975; 57-A(2):259-261.

[9] Sobera M, Siedlecka B, Syczewska M. Posture control development in children aged 2-7 years old, based on the changes of repeatability of the stability indices. Neurosci Lett 2011; 491(1):13-7.

[10] Staheli LT. Rotational problems in children. J Bone Joint Surg 1993; 75A(6):939-949.

[11] Pfeffier M, Kotz R, Ledl T, Hauser G, Sluga M. Prevalence of flat-foot in preschool children. Paediatrics 2006; 118(2):643-649.

[12] Sutherland D. The development of mature gait. Gait Posture 1997; 6(2);163170. 
[13] Vanderwilde R, Staheli L, Chew DE, Malagon V. Measurements on radiographs of the foot in normal infants and children. J Bone Joint Surg Am 1988; 70(3):407415.

[14] Chester VL, Tingley M, Edmund NB. A comparison of kinetic gait parameters for 3-13 year olds. Clin Biomech 2006; 21(7):726-732.

[15] Cupp T, Oeffinger D, Tylkowski C, Augsburger S. Age-related kinetic changes in normal pediatrics. J Ped Orthop 1999; 19(4):475-478.

[16] Lundgren, P, C Nester, A Liu, A, Arndt, R Jones, Stacoff, A, Wolf, P. Lundberg, A. Invasive in vivo measurement of rear, mid and forefoot motion during walking. Gait Posture 2008; 28(1):93-100.

[17] Pataky TC, Goulermas JY. Pedobarographic statistical parametric mapping (pSPM): A pixel-level approach to foot pressure image analysis. J Biomech 2008; 41: $2136-2143$.

[18] Pataky TC. Spatial resolution in plantar pressure measurement revisited. J Biomech 2012; 45(12):2116-2124.

[19] Lord M. Spatial resolution in plantar pressure measurement. Med Eng Phys 1997; 19(2):140-144. 
[20] Phethean J, Nester CJ. The influence of body weight, body mass index and gender on plantar pressures: Results of a cross-sectional study of healthy children's feet. Gait Posture 2012; 36:287-290.

[21] Bellman M, Longman S, Aukett A. Schedule of Growing Skills in Practice. Windsor:NFER-Nelson; 1997.

[22] Freeman JV, Cole TJ, Chinn S, Jones PRM, White EM, Preece MA. Cross sectional stature and weight reference curves for the UK, 1990. Arch Dis Child 1995; 73:17-24.

[23] Oladeji PT, Stackhouse C, Gracely E, Orlin M. Comparison of the two-step and midgait methods in children. J Am Podiatr Med Assoc 2008; 98(4): 268-277.

[24] Cavanagh PR, Ulbrecht JS. Clinical plantar pressure measurement in diabetes: rationale and methodology. The Foot 1994; 4:123-135.

[25] Oliveira FPM, Pataky TC, Tavares JMRS. Registration of pedobarographic image data in frequency domain. Computer Methods in Biomechanics and Biomedical Engineering 2010; 13(6): 262-269.

[26] Friston KJ, Ashburner JT, Kiebel SJ, Nichols TE, Penny WD, Eds. Statistical Parametric Mapping: The Analysis of Functional Brain Images. London: Elsevier; 2007. 
[27] Pataky TC. Assessing the significance of pedobarographic signals using random field theory. J Biomech 2008; 41(11):2465-2473.

[28] Giacomozzi C, Macallari V, Leardini A, Benedetti MG. Integrated pressureforce-kinematics measuring system for the characterisation of plantar foot loading during locomotion. Med Biol Eng Comput 2000; 38:156-63.

[29] Stebbins J, Giacomozzi C, Theologis T. Correlation between plantar pressure and Oxford Foot Model kineamtics. J Foot Ankle Res 2008; 1:O22.

[30] Keijsers N, Stolwijk N, Pataky TC. Linear dependence of peak, mean, and pressure-time integral values in plantar pressure images. Gait Posture 2010; 31 : $140-142$.

[31] Pataky TC, Caravaggi P, Savage R, Crompton, R. Regional peak plantar pressures are highly sensitive to region boundary definitions. J Biomech 2008; $41: 2772-2775$.

[32] Lin C-J, Lai K-A, Kuan T-S, Chou Y-L. Correlating factors and clinical significance of flexible flatfoot in preschool children. J Ped Orthop 2001; 21:378-382.

[33] Nester CJ, Liu AM, Ward E, Howard D, Cocheba J, Derrick T, Patterson P. In vitro study of foot kinematics using a dynamic walking simulator. J Biomech 2007; 40(9):1927-1937. 
Table 1. Barefoot peak plantar pressures for children aged 4-7 years $(\mathrm{kPa})$.

\begin{tabular}{|c|c|c|c|c|c|c|c|c|c|c|c|c|c|}
\hline & $\begin{array}{l}\text { Current } \\
\text { study }\end{array}$ & $\begin{array}{l}\text { Bosch et } \\
\text { al, } 2010\end{array}$ & $\begin{array}{l}\text { Müller et } \\
\text { al, } 2011\end{array}$ & $\begin{array}{l}\text { Current } \\
\text { study }\end{array}$ & $\begin{array}{c}\text { Bosch et } \\
\text { al, } 2010\end{array}$ & $\begin{array}{l}\text { Müller et } \\
\text { al, } 2011\end{array}$ & $\begin{array}{l}\text { Current } \\
\text { study }\end{array}$ & $\begin{array}{l}\text { Bosch et } \\
\text { al, } 2010\end{array}$ & $\begin{array}{l}\text { Müller et } \\
\text { al, } 2011\end{array}$ & $\begin{array}{l}\text { Current } \\
\text { study }\end{array}$ & $\begin{array}{l}\text { Bosch et } \\
\text { al, } 2010\end{array}$ & $\begin{array}{l}\text { Müller et } \\
\text { al, } 2011\end{array}$ & $\begin{array}{l}\text { Bosch et } \\
\text { al, } 2009\end{array}$ \\
\hline & 4 years & 4.2 years & 4 years & 5 years & 5.2 year & 5 years & 6 years & 6.2 years & 6 years & 7 years & 7.2 years & 7 years & 7 years \\
\hline & mean & median & mean & mean & median & mean & mean & median & mean & mean & median & mean & mean \\
\hline Heel & 258 & 213 & 267 & 280 & 246 & 280 & 293 & 359 & 286 & 287 & 370 & 301 & 384 \\
\hline MedMF & 22 & \multirow{2}{*}{67} & \multirow{2}{*}{87} & 17 & \multirow{2}{*}{64} & \multirow{2}{*}{82} & 28 & \multirow{2}{*}{74} & \multirow{2}{*}{80} & 31 & \multirow{2}{*}{70} & \multirow{2}{*}{77} & \multirow{2}{*}{83} \\
\hline LatMF & 50 & & & 43 & & & 48 & & & 48 & & & \\
\hline MH1 & 134 & \multirow{5}{*}{154} & \multirow{5}{*}{159} & 162 & \multirow{5}{*}{184} & \multirow{5}{*}{175} & 147 & \multirow{5}{*}{231} & \multirow{5}{*}{188} & 172 & \multirow{5}{*}{247} & \multirow{5}{*}{208} & \multirow{5}{*}{256} \\
\hline MH2 & 156 & & & 145 & & & 185 & & & 198 & & & \\
\hline MH3 & 132 & & & 148 & & & 160 & & & 186 & & & \\
\hline MH4 & 109 & & & 116 & & & 116 & & & 122 & & & \\
\hline MH5 & 80 & & & 90 & & & 96 & & & 80 & & & \\
\hline Hallux & 223 & 164 & & 224 & 191 & & 217 & 251 & & 216 & 282 & & 272 \\
\hline
\end{tabular}


(a)

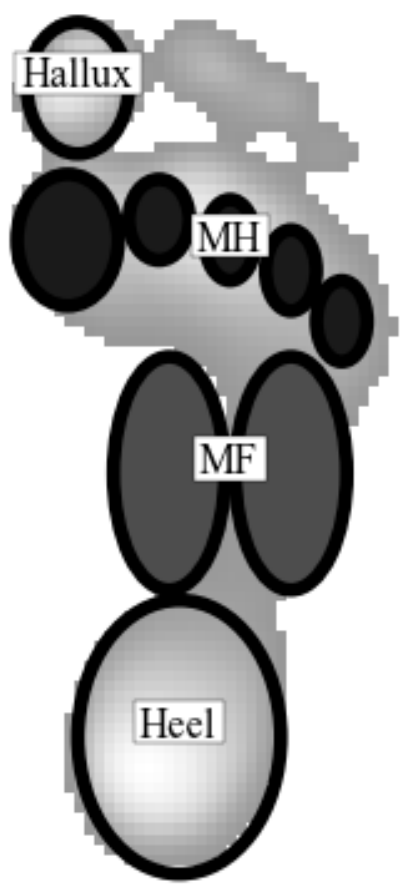

(b)

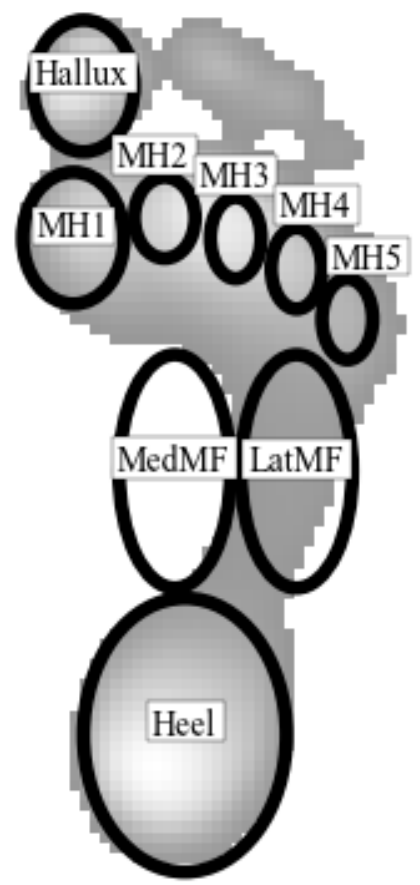

Figure 1: Region of interest (ROI) definitions: (a) Method A, and (b) Method B. 


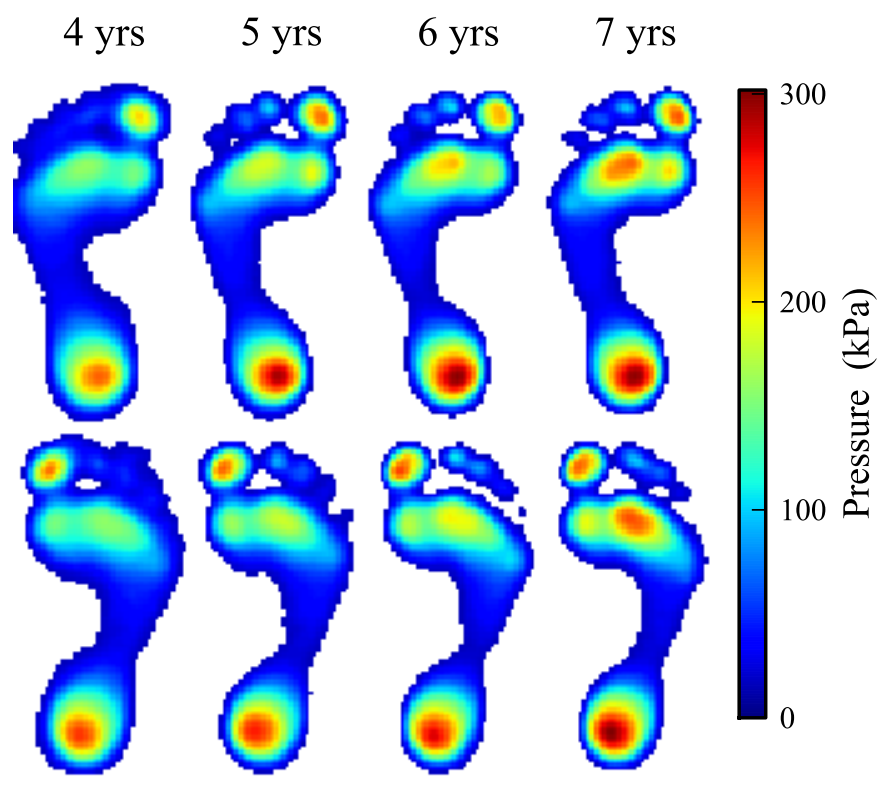

Figure 2: Average peak pressure distributions for the four age groups. 

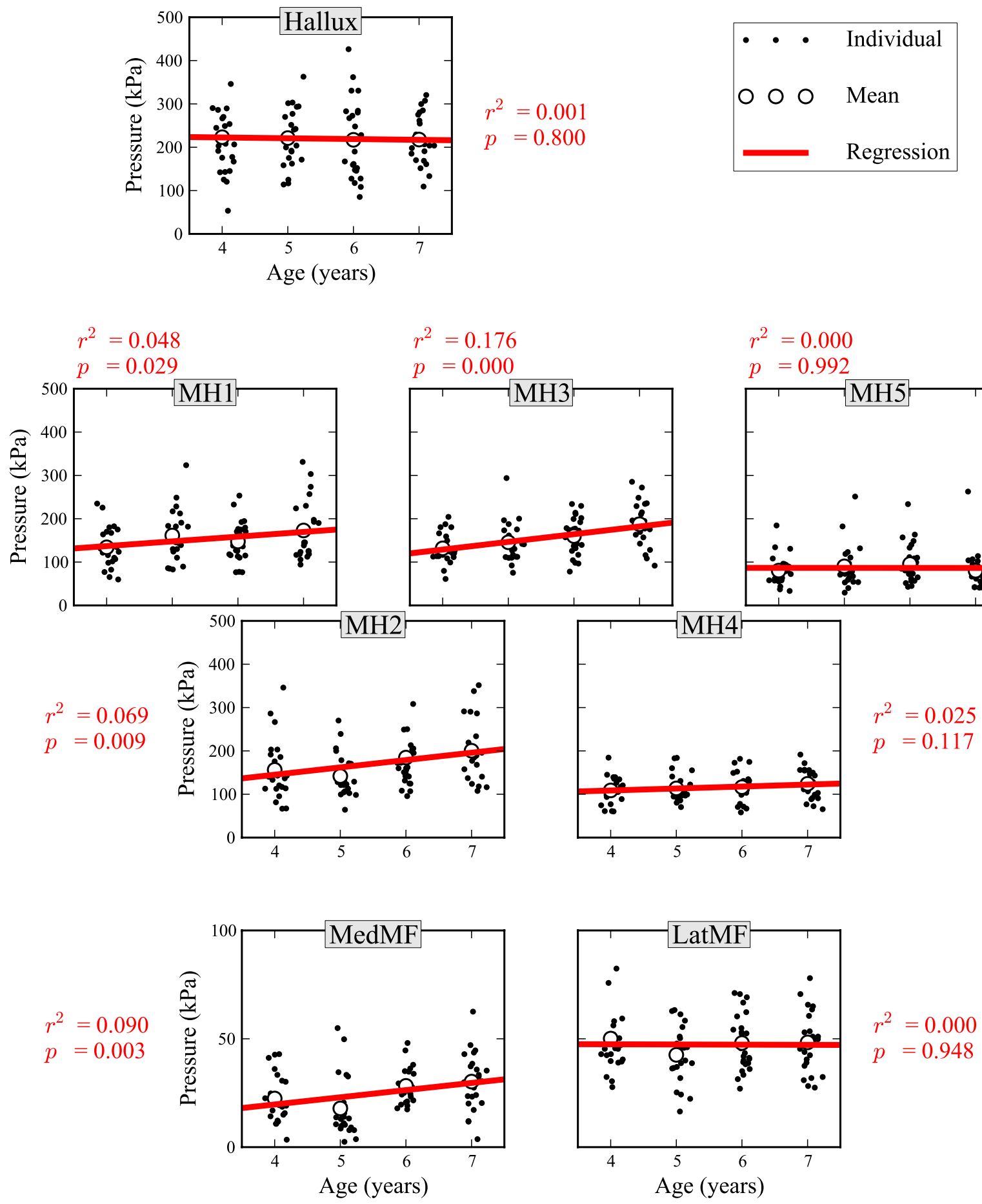

$r^{2}=0.025$
$p=0.117$
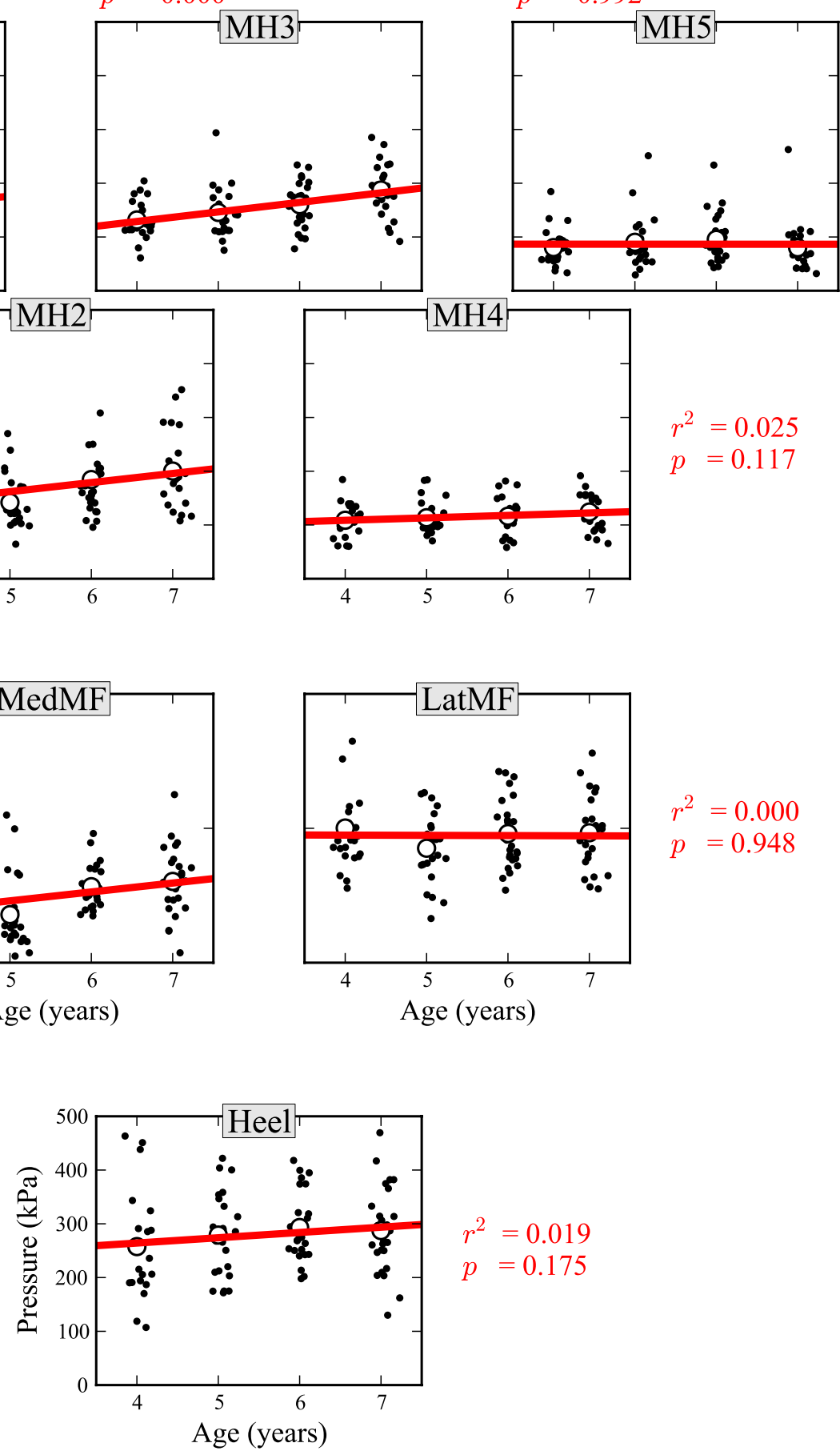

Figure 3: Linear regression results for the region of interest (ROI) data (left foot). 
(a) Raw SPM

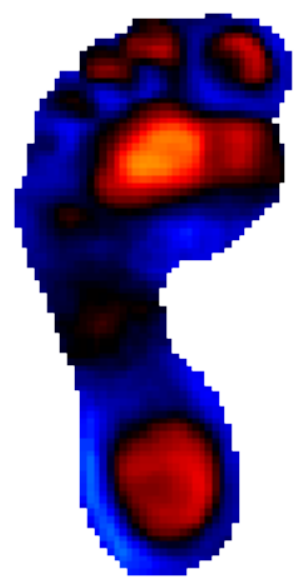

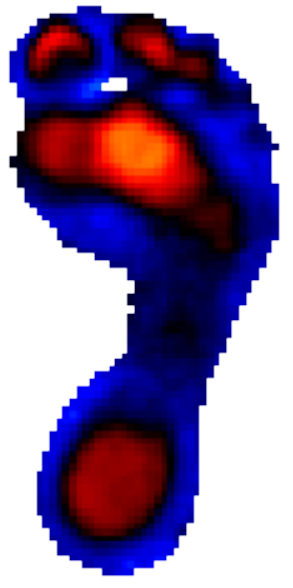

(b) Inference
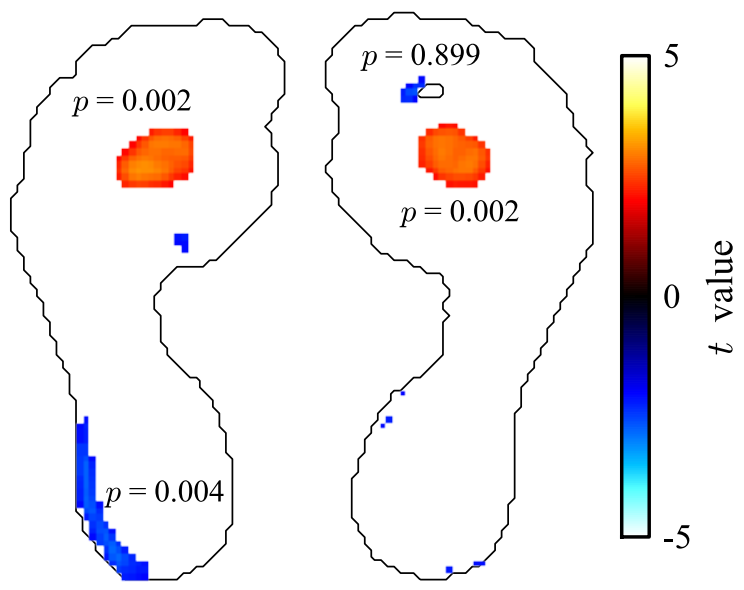

Figure 4: Linear regression results for the pixel-level data. (a) The raw statistical parametric map (SPM) was computed by conducting separate linear regression at all pixels; $t$ values indicate the strength of linear regression (Eqn.1). (b) Inference images, depicting the portions of the feet which reached (or failed to reach) statistical significance; $|t|>2$. 
SUPPLEMENTARY 

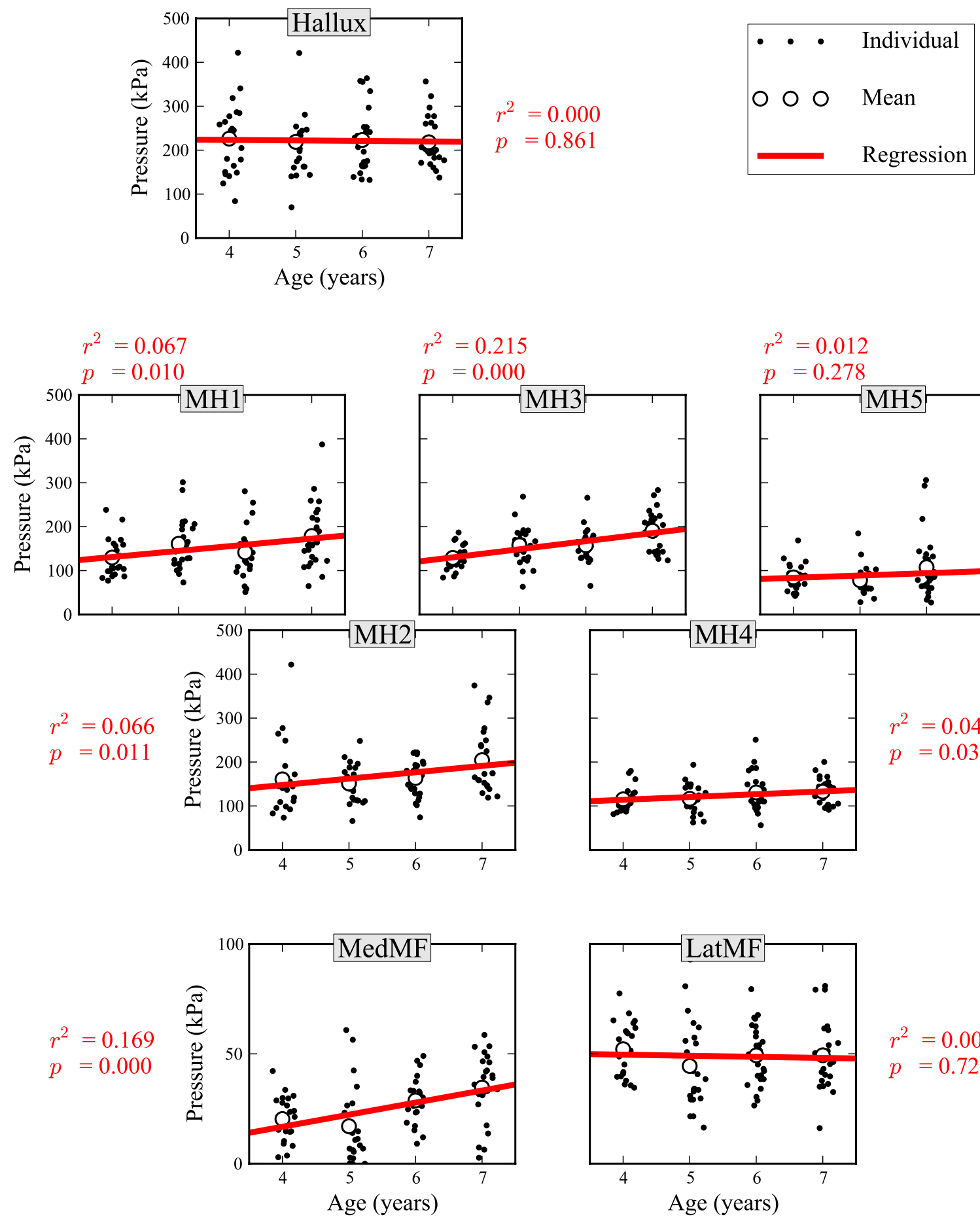

$$
\begin{aligned}
& r^{2}=0.012 \\
& p=0.278
\end{aligned}
$$

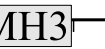

